\title{
花生籽仁庶糖含量近红外模型构建及在高糖品种培育中的应用
}

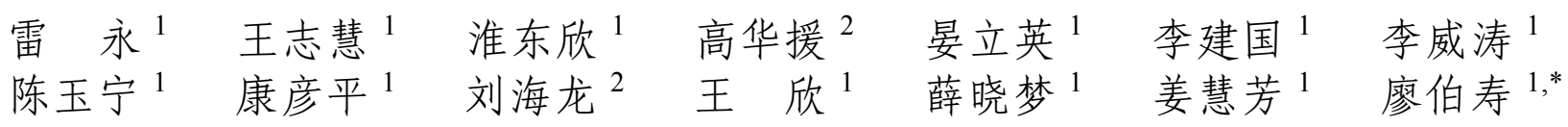

1 中国农业科学院油料作物研究所 / 农业农村部油料作物生物学重点开放实验室, 湖北武汉 $430062 ;^{2}$ 吉林省农业科学院花生研究 所, 吉林公主岭 136100

摘 要: 含糖量是决定和影响花生食用品质和加工特性的重要指标，蔗糖含量占成熟花生籽仁总糖量的 $90 \%$ 以上， 建立蔗糖含量的高效检测技术, 有助于加快高蔗糖甜味食用型花生品种培育进程。本研究利用蔗糖含量差异显著的 185 份花生材料, 利用近红外仪(波长范围 1100 2500 nm), 配合小样品杯, 扫描和采集自然干燥籽仁的近红外光谱, 采用液相色谱(HPLC)结合标准曲线法测定试验材料的蔗糖含量, 利用偏最小二乘法(partial least squares, PLS)构建了 花生籽仁熫糖含量的近红外定标模型, 模型的决定系数 $R^{2}=0.962$, 均方差为 0.383 。利用 20 份材料对模型进行外部 验证, 预测值和化学值的决定系数达 0.947 , 表明该模型可较好地预测蔗糖含量, 可以高效地测定杂交早期世代的单 株花生蔗糖含量。利用该模型在“吉花 02-1-4×中花 26” 杂交后代中发掘出 6 份含糖量 7\%以上、油酸 78\%以上、含油 量 48\%以下，且农艺性状优良的食用花生新品系。

关键词: 花生; 蔗糖含量; 近红外模型; 高庶糖; 品种

\section{Development and application of a near infrared spectroscopy model for predicting high sucrose content of peanut seed}

LEI Yong ${ }^{1}$, WANG Zhi-Hui ${ }^{1}$, HUAI Dong-Xin ${ }^{1}$, GAO Hua-Yuan ${ }^{2}$, YAN Li-Ying ${ }^{1}$, LI Jian-Guo ${ }^{1}$, LI Wei-Tao ${ }^{1}$, CHEN Yu-Ning ${ }^{1}$, KANG Yan-Ping ${ }^{1}$, LIU Hai-Long ${ }^{2}$, WANG Xin ${ }^{1}$, XUE Xiao-Meng ${ }^{1}$, JIANG Hui-Fang ${ }^{1}$, and LIAO Bo-Shou ${ }^{1, *}$

${ }^{1}$ Oil Crops Research Institute, Chinese Academy of Agricultural Sciences / Key Laboratory of Oil Crop Biology of the Ministry of Agriculture and Rural Affairs, Wuhan 430062, Hubei, China; ${ }^{2}$ Peanut Research Institute, Jilin Academy of Agricultural Sciences, Gongzhuling 136100, Jilin, China

\begin{abstract}
Sugar content is an important factor affecting the flavor and processing characteristics of peanut (Arachis hypogaea L.) kernel and the sucrose content accounts for more than $90 \%$ of the total sugar content in peanut kernel. High-efficiency detection approach for sucrose content is crucial in developing high sucrose peanut varieties. In this study, 185 peanut genotypes with diversified sucrose contents were scanned in wave length of 1100-2500 nm for constructing a near-infrared spectrum of naturally dried seeds. The sucrose contents were determined by high performance liquid chromatography (HPLC) combined with standard curve method. A near-infrared calibration model of peanut seed sucrose content was established by partial least square method (PLS). For sucrose content, the coefficient of determinations $\left(R^{2}\right)$ was 0.962 with mean square deviation of 0.383 . The coefficient of correlation between the predicted values and chemically tested value were 0.947 in 20 external samples, indicating that the model could predict sucrose content of peanut kernel. Six new sweet peanut lines with sucrose content over 7\%, oleic acid over $78 \%$, oil content less than $48 \%$ and desirable agronomic characters were selected among the hybrid progenies of "Jihua 02-1-4 $\times$ Zhonghua 26 ".
\end{abstract}

\footnotetext{
本研究由国家重点研发计划项目(2018YFD1000900), 农业农村部油料作物生物学与遗传育种重点实验室开放课题(KF2020008), 中国 农业科学院科技创新工程(CAAS-ASTIP-2013-OCRI)和广东省重点领域研发计划项目(2020B020219003)资助。

This study was supported by the National Key Research and Development Program of China (2018YFD1000900), the Open Project of Key Laboratory of Biology and Genetic Improvement of Oil Crops, Ministry of Agriculture and Rural Affairs (KF2020008), the Innovation Program of the Chinese Academy of Agricultural Sciences (CAAS-ASTIP-2013-OCRI), and Key-Area Research and Development Program of Guangdong Province (2020B020219003).
}

*通信作者(Corresponding author): 廖伯寿, E-mail: 1boshou@hotmail.com

第一作者联系方式: E-mail: leiyong@caas.cn

Received (收稿日期): 2020-05-14; Accepted (接受日期): 2020-08-19; Published online (网络出版日期): 2020-09-21.

URL: https://kns.cnki.net/kcms/detail/11.1809.S.20200921.1535.005.html 
Keywords: peanut; sucrose content; near infrared spectroscopy (NIRS) model; high sucrose content; varieties

花生是我国重要的油料和经济作物。近 10 年来, 我国花生年均总产约 1680 万吨, 居世界首位, 占全 球花生总产的 $38 \%^{[1]}$ 。我国花生总产约 $50 \%$ 用于榨 油, 40\%作为食用，欧美等发达国家生产和进口的花 生则 $70 \%$ 以上作为食用和食品加工原料。随着人民 生活水平的提高和加工技术的进步, 我国花生食用 及食品加工量将越来越大, 对原料的需求越来越多 样化 ${ }^{[2]}$ 。含糖量高低对于花生的食用品质、加工风 味有重要影响, 含糖量和甜度高是鲜食花生的关键 品质指标。研究表明, 花生籽仁含糖量可作为预测 烤花生风味和甜度的重要指标, 与花生籽仁口味品 质相关系数达 0.88 , 糖含量的微小变化也会影响最终 的烘烤质量 ${ }^{[3-5]}$ 。花生干籽仁中蔗糖含量达到 $6 \%$ 以上 时, 口感较好。成熟花生籽仁中的糖类主要是蔗糖, 占 $90 \%$ 左右。因此, 发掘和培育高庶糖含量的种质和 品种, 对于食用型花生品质的提升具有重要价值。

已有研究表明, 花生含糖量是可以遗传的性状 ${ }^{[3-6]}$, 不同市场型花生的含糖量有显著差异, 兰娜型 (runner type)、弗吉尼亚型(virginia type)、瓦伦西亚 型(valencia type)的总糖量均值分别为 $2.90 \% 、 3.51 \%$ 和 $2.88 \%$, 西班牙型(spanish type)花生资源的蔗糖含 量在 $2.44 \% \sim 7.61 \%$ 之间 ${ }^{[7-8]}$ 。如何对资源材料和育种 后代的蔗糖含量进行高通量、规模化的检测分析, 是选育高糖优异食用型花生品种的关键技术。目前, 传统的花生籽仁庶糖含量测定方法多采用比色法和 液相色谱法, 耗时、费力且不能进行高通量检测。
近红外光谱法具有非破坏性、快速、高效的特点, 在农 作物品质分析中已得到广泛应用 ${ }^{[9-11]}$ 。花生中已经建立 了含油量、脂肪酸、蛋白质、氨基酸等重要品质指标的 近红外模型, 但在籽仁蔗糖检测方面应用的较少 ${ }^{[12-19]}$ 。 秦利等 ${ }^{[20]}$ 和唐月异等 ${ }^{[21]}$ 分别利用瑞典波通 DA7200 和 德国布鲁克 Matris-I 近红外光谱仪建立了多粒花生籽 仁的蔗糖含量近红外模型, 但建立模型与测试时需要 的籽仁量较大(30 50 粒), 不能对籽仁量较少的单株进 行蔗糖含量检测, 并且以上 2 种型号光谱仪采集的光 谱无法在其他公司生产的光谱仪上应用 ${ }^{[20-21]}$ 。

为了深入探索近红外光谱分析法的仪器广适性, 本研究通过对 185 份花生样品熫糖含量的测定, 利用 Unity 近红外仪扫描样品的光谱, 建立和优化花生成 熟籽仁蔗糖含量的近红外分析模型, 以期为花生熫 糖含量测定提供更加快捷有效的方法, 加快食用型 花生品种选育进程。

\section{1 材料与方法}

\section{1 研究材料}

建模所用花生材料系本团队收集的国内育成品 种和培育的高含糖量中间材料, 总计 185 份, 其中 S001 S121 是团队选育的蔗糖含量较高的育种中间 材料, 种植地点为湖北武汉, S122 S185 是䇻选的含 糖量有显著差异的品种在多个地方种植的样品(表 1)。另取 20 份样品, 编号为 ST01 ST20, 作为外部 样品用于模型验证。

表 1 建模和模型验证所用花生品种(系)编号及名称

Table 1 Peanut accessions used for developing NIR model

\begin{tabular}{ccc||ccc}
\hline $\begin{array}{c}\text { 样品编号 } \\
\text { Sample ID }\end{array}$ & $\begin{array}{c}\text { 品种(系) } \\
\text { Variety (line) }\end{array}$ & $\begin{array}{c}\text { 种植地点 } \\
\text { Location }\end{array}$ & $\begin{array}{c}\text { 样品编号 } \\
\text { Sample ID }\end{array}$ & $\begin{array}{c}\text { 品种(系) } \\
\text { Variety (line) }\end{array}$ & $\begin{array}{c}\text { 种植地点 } \\
\text { Location }\end{array}$ \\
\hline S001 & A1435-3 & 湖北武汉 Wuhan, Hubei & S104 & A1516-1 & 湖北武汉 Wuhan, Hubei \\
S002 & A1435-4 & 湖北武汉 Wuhan, Hubei & S105 & A1516-2 & 湖北武汉 Wuhan, Hubei \\
S003 & A1442-1 & 湖北武汉 Wuhan, Hubei & S106 & A1518-1 & 湖北武汉 Wuhan, Hubei \\
S004 & A1444-1 & 湖北武汉 Wuhan, Hubei & S107 & A1518-2 & 湖北武汉 Wuhan, Hubei \\
S005 & A1444-2 & 湖北武汉 Wuhan, Hubei & S108 & A1520-1 & 湖北武汉 Wuhan, Hubei \\
S006 & A1444-4 & 湖北武汉 Wuhan, Hubei & S109 & A1520-2 & 湖北武汉 Wuhan, Hubei \\
S007 & A1444-5 & 湖北武汉 Wuhan, Hubei & S110 & A1529-1 & 湖北武汉 Wuhan, Hubei \\
S008 & A1444-6 & 湖北武汉 Wuhan, Hubei & S111 & A1529-2 & 湖北武汉 Wuhan, Hubei \\
S009 & A1447-1 & 湖北武汉 Wuhan, Hubei & S112 & A1532 & 湖北武汉 Wuhan, Hubei \\
S010 & A1447-2 & 湖北武汉 Wuhan, Hubei & S113 & A1538 & 湖北武汉 Wuhan, Hubei \\
S011 & A1447-3 & 湖北武汉 Wuhan, Hubei & S114 & A1541 & 湖北武汉 Wuhan, Hubei \\
S012 & A1447-4 & 湖北武汉 Wuhan, Hubei & S115 & A1543-1 & 湖北武汉 Wuhan, Hubei \\
S013 & A1447-5 & 湖北武汉 Wuhan, Hubei & S116 & A1543-2 & 湖北武汉 Wuhan, Hubei \\
\hline
\end{tabular}




\begin{tabular}{|c|c|c|c|c|c|c|}
\hline $\begin{array}{l}\text { 样品编号 } \\
\text { Sample ID }\end{array}$ & $\begin{array}{c}\text { 品种(系) } \\
\text { Variety (line) }\end{array}$ & $\begin{array}{c}\text { 种植地点 } \\
\text { Location }\end{array}$ & $\begin{array}{l}\text { 样品编号 } \\
\text { Sample ID }\end{array}$ & $\begin{array}{c}\text { 品种(系) } \\
\text { Variety (line) }\end{array}$ & & $\begin{array}{c}\text { 种植地点 } \\
\text { Location }\end{array}$ \\
\hline S014 & A1447-7 & 湖北武汉 Wuhan, Hubei & S117 & A1547-1 & 湖北武汉 & Wuhan, Hubei \\
\hline S015 & A1449-1 & 湖北武汉 Wuhan, Hubei & $\mathrm{S} 118$ & A1547-2 & 湖北武汉 & Wuhan, Hubei \\
\hline S016 & A1449-2 & 湖北武汉 Wuhan, Hubei & S119 & A1547-3 & 湖北武汉 & Wuhan, Hubei \\
\hline S017 & A1449-3 & 湖北武汉 Wuhan, Hubei & $\mathrm{S} 120$ & A1547-4 & 湖北武汉 & Wuhan, Hubei \\
\hline S018 & A1449-4 & 湖北武汉 Wuhan, Hubei & $\mathrm{S} 121$ & A1547-5 & 湖北武汉 & Wuhan, Hubei \\
\hline S019 & A1449-5 & 湖北武汉 Wuhan, Hubei & $\mathrm{S} 122$ & 花育 23 Huayu 23 & 湖北武汉 & Wuhan, Hubei \\
\hline S020 & A1449-6 & 湖北武汉 Wuhan, Hubei & $\mathrm{S} 123$ & 远杂 9102 Yuanza 9102 & 湖北武汉 & Wuhan, Hubei \\
\hline S021 & A1449-7 & 湖北武汉 Wuhan, Hubei & $\mathrm{S} 124$ & 锦花 15 Jinhua 15 & 湖北武汉 & Wuhan, Hubei \\
\hline S022 & A1449-8 & 湖北武汉 Wuhan, Hubei & $\mathrm{S} 125$ & 贵州红 Guizhouhong & 湖北武汉 & Wuhan, Hubei \\
\hline S023 & A1449-10 & 湖北武汉 Wuhan, Hubei & $\mathrm{S} 126$ & 扶余四粒红 Fuyusilihong & 湖北武汉 & Wuhan, Hubei \\
\hline S024 & A1449-11 & 湖北武汉 Wuhan, Hubei & S127 & 花育 16 Huayu 16 & 湖北武汉 & Wuhan, Hubei \\
\hline S025 & A $1449-12$ & 湖北武汉 Wuhan, Hubei & $\mathrm{S} 128$ & 云花 3 号 Yunhua 3 & 湖北武汉 & Wuhan, Hubei \\
\hline S026 & A1449-13 & 湖北武汉 Wuhan, Hubei & S129 & 山东仔 Shandongzai & 湖北武汉 & Wuhan, Hubei \\
\hline S027 & A1449-14 & 湖北武汉 Wuhan, Hubei & $\mathrm{S} 130$ & 麻阳小子 Mayangxiaozi & 湖北武汉 & Wuhan, Hubei \\
\hline S028 & A1454-1 & 湖北武汉 Wuhan, Hubei & S131 & 扶余四粒红 Fuyusiliong & 湖北武汉 & Wuhan, Hubei \\
\hline S029 & A1454-2 & 湖北武汉 Wuhan, Hubei & $\mathrm{S} 132$ & 青花 505 Qinhua 505 & 湖北武汉 & Wuhan, Hubei \\
\hline S030 & A1454-3 & 湖北武汉 Wuhan, Hubei & S133 & 赣花 7 号 Ganhua 7 & 湖北武汉 & Wuhan, Hubei \\
\hline S031 & A1454-4 & 湖北武汉 Wuhan, Hubei & $\mathrm{S} 134$ & 红豆花生 Hongdouhuasheng & 湖北武汉 & Wuhan, Hubei \\
\hline S032 & A1454-5 & 湖北武汉 Wuhan, Hubei & $\mathrm{S} 135$ & 团风小红粒 Tuanfengxiaoli & 湖北武汉 & Wuhan, Hubei \\
\hline S033 & A1454-6 & 湖北武汉 Wuhan, Hubei & $\mathrm{S} 136$ & 沿河本地种 Yanhebendizhong & 湖北武汉 & Wuhan, Hubei \\
\hline S034 & A1458-1 & 湖北武汉 Wuhan, Hubei & S137 & 贵州红花生 Guizhouhonghuasheng & 湖北武汉 & Wuhan, Hubei \\
\hline S035 & A1458-3 & 湖北武汉 Wuhan, Hubei & $\mathrm{S} 138$ & 贵州红皮 Guizhouhongpi & 湖北武汉 & Wuhan, Hubei \\
\hline S036 & A1458-4 & 湖北武汉 Wuhan, Hubei & S139 & 红花生 Honghuasheng & 湖北武汉 & Wuhan, Hubei \\
\hline S037 & A1463 & 湖北武汉 Wuhan, Hubei & $\mathrm{S} 140$ & $14-511855$ & 湖北武汉 & Wuhan, Hubei \\
\hline S038 & A1464-1 & 湖北武汉 Wuhan, Hubei & S141 & 皖花 2 号 Wanhua 2 & 湖北武汉 & Wuhan, Hubei \\
\hline S039 & A1464-2 & 湖北武汉 Wuhan, Hubei & $\mathrm{S} 142$ & 日照瓜子 Rizhaoguazi & 湖北武汉 & Wuhan, Hubei \\
\hline S040 & A1464-3 & 湖北武汉 Wuhan, Hubei & S143 & 天府 3 号 Tianfu 3 & 湖北武汉 & Wuhan, Hubei \\
\hline S041 & A1464-4 & 湖北武汉 Wuhan, Hubei & $\mathrm{S} 144$ & 天府 22 Tianfu 22 & 湖北武汉 & Wuhan, Hubei \\
\hline S042 & A1464-5 & 湖北武汉 Wuhan, Hubei & $\mathrm{S} 145$ & 开封水果 Kaifengshuiguo & 湖北武汉 & Wuhan, Hubei \\
\hline S043 & A1464-6 & 湖北武汉 Wuhan, Hubei & S146 & 冀花 10 号 Jihua 10 & 湖北武汉 & Wuhan, Hubei \\
\hline S044 & A1464-7 & 湖北武汉 Wuhan, Hubei & $\mathrm{S} 147$ & 河北水果 Hebeishuiguo & 湖北武汉 & Wuhan, Hubei \\
\hline S045 & A1467-1 & 湖北武汉 Wuhan, Hubei & $\mathrm{S} 148$ & 红仁花生 Hongrenhuasheng & 湖北武汉 & Wuhan, Hubei \\
\hline S046 & A1467-2 & 湖北武汉 Wuhan, Hubei & S149 & 四粒红 Silihong & 湖北武汉 & Wuhan, Hubei \\
\hline S047 & A1467-3 & 湖北武汉 Wuhan, Hubei & $\mathrm{S} 150$ & 杜皮红 Dupihong & 湖北武汉 & Wuhan, Hubei \\
\hline S048 & A1467-4 & 湖北武汉 Wuhan, Hubei & $\mathrm{S} 151$ & 钟山红豆 Zhongshanhongdou & 湖北武汉 & Wuhan, Hubei \\
\hline S049 & A1467-5 & 湖北武汉 Wuhan, Hubei & $\mathrm{S} 152$ & 吉花 18 Jihua 18 & 湖北武汉 & Wuhan, Hubei \\
\hline S050 & A1467-6 & 湖北武汉 Wuhan, Hubei & $\mathrm{S} 153$ & 吉花 19 Jihua 19 & 湖北武汉 & Wuhan, Hubei \\
\hline $\mathrm{S} 051$ & A1471-1 & 湖北武汉 Wuhan, Hubei & $\mathrm{S} 154$ & 吉花 20 Jihua 20 & 湖北武汉 & Wuhan, Hubei \\
\hline S052 & A1471-2 & 湖北武汉 Wuhan, Hubei & S155 & 吉花 23 Jihua 23 & 湖北武汉 & Wuhan, Hubei \\
\hline S053 & A1471-3 & 湖北武汉 Wuhan, Hubei & S156 & 吉花 24 Jihua 24 & 湖北武汉 & Wuhan, Hubei \\
\hline S054 & A1471-4 & 湖北武汉 Wuhan, Hubei & $\mathrm{S} 157$ & 阜花 25 Fuhua 25 & 湖北武汉 & Wuhan, Hubei \\
\hline S055 & A $1473-1$ & 湖北武汉 Wuhan, Hubei & $\mathrm{S} 158$ & 阜花 26 Fuhua 26 & 湖北武汉 & Wuhan, Hubei \\
\hline S056 & A1473-2 & 湖北武汉 Wuhan, Hubei & S159 & 阜花 30 Fuhua 30 & 湖北武汉 & Wuhan, Hubei \\
\hline S057 & A1473-3 & 湖北武汉 Wuhan, Hubei & $\mathrm{S} 160$ & 花育 23 Huayu 23 & 安徽合肥 & Hefei, Anhui \\
\hline S058 & A1473-4 & 湖北武汉 Wuhan, Hubei & $\mathrm{S} 161$ & 四粒红 Silihong & 河北石家 & 庄 Shijiazhuang, Hebei \\
\hline
\end{tabular}


(续表 1)

\begin{tabular}{|c|c|c|c|c|c|}
\hline $\begin{array}{l}\text { 样品编号 } \\
\text { Sample ID }\end{array}$ & $\begin{array}{c}\text { 品种(系) } \\
\text { Variety (line) }\end{array}$ & $\begin{array}{c}\text { 种植地点 } \\
\text { Location }\end{array}$ & $\begin{array}{l}\text { 样品编号 } \\
\text { Sample ID }\end{array}$ & $\begin{array}{c}\text { 品种(系) } \\
\text { Variety (line) }\end{array}$ & $\begin{array}{l}\text { 种植地点 } \\
\text { Location }\end{array}$ \\
\hline S059 & A1473-5 & 湖北武汉 Wuhan, Hubei & S162 & 冀 113 Ji 113 & 河北石家庄 Shijiazhuang, Hebei \\
\hline S060 & A1473-6 & 湖北武汉 Wuhan, Hubei & S163 & 远杂 9102 Yuanza 9102 & 河北石家庄 Shijiazhuang, Hebei \\
\hline S061 & A1473-7 & 湖北武汉 Wuhan, Hubei & S164 & 冀 11 Ji 11 & 河北石家庄 Shijiazhuang, Hebei \\
\hline S062 & A1473-8 & 湖北武汉 Wuhan, Hubei & S165 & 冀花 18 Jihua 18 & 河北石家庄 Shijiazhuang, Hebei \\
\hline S063 & A1476 & 湖北武汉 Wuhan, Hubei & S166 & 冀甜 1 号 Jitian 1 & 河北石家庄 Shijiazhuang, Hebei \\
\hline S064 & A1478-1 & 湖北武汉 Wuhan, Hubei & S167 & 中花 16 Zhonghua 16 & 四川成都 Chengdu, Sichuan \\
\hline S065 & A1480-1 & 湖北武汉 Wuhan, Hubei & S168 & 蜀花 3 号 Shuhua 3 & 四川成都 Chengdu, Sichuan \\
\hline S066 & A $1480-2$ & 湖北武汉 Wuhan, Hubei & S169 & 天府 11 Tianfu 11 & 四川成都 Chengdu, Sichuan \\
\hline S067 & A $1480-3$ & 湖北武汉 Wuhan, Hubei & $\mathrm{S} 170$ & 花育 23 Huayu 23 & 四川成都 Chengdu, Sichuan \\
\hline S068 & A1480-4 & 湖北武汉 Wuhan, Hubei & S171 & 远杂 9102 Yuanza 9102 & 四川成都 Chengdu, Sichuan \\
\hline S069 & A1483-1 & 湖北武汉 Wuhan, Hubei & S172 & 中花 6 号 Zhonghua 16 & 四川成都 Chengdu, Sichuan \\
\hline S070 & A $1483-2$ & 湖北武汉 Wuhan, Hubei & S173 & 四粒红 Silihong & 四川成都 Chengdu, Sichuan \\
\hline S071 & A1483-3 & 湖北武汉 Wuhan, Hubei & S174 & 中花 24 Zhonghua 24 & 四川成都 Chengdu, Sichuan \\
\hline S072 & A1485-1 & 湖北武汉 Wuhan, Hubei & S175 & 中花 21 Zhonghua 21 & 四川成都 Chengdu, Sichuan \\
\hline S073 & A $1485-2$ & 湖北武汉 Wuhan, Hubei & S176 & 罗汉果 Luohanguo & 四川成都 Chengdu, Sichuan \\
\hline S074 & A $1485-3$ & 湖北武汉 Wuhan, Hubei & S177 & 中花 21 Zhonghua 21 & 安徽合肥 Hefei, Anhui \\
\hline S075 & A1485-4 & 湖北武汉 Wuhan, Hubei & S178 & 罗汉果 Luohanguo & 安徽合肥 Hefei, Anhui \\
\hline S076 & A1488 & 湖北武汉 Wuhan, Hubei & S179 & 中花 215 Zhonghua 215 & 安徽合肥 Hefei, Anhui \\
\hline S077 & A1490-1 & 湖北武汉 Wuhan, Hubei & S180 & 中花 16 Zhonghua 16 & 安徽合肥 Hefei, Anhui \\
\hline S078 & A1490-2 & 湖北武汉 Wuhan, Hubei & $\mathrm{S} 181$ & 四粒红 Silihong & 安徽合肥 Hefei, Anhui \\
\hline S079 & A1493-1 & 湖北武汉 Wuhan, Hubei & S182 & 中花 21 Zhonghua 21 & 河北石家庄 Shijiazhuang, Hebei \\
\hline S080 & A1493-2 & 湖北武汉 Wuhan, Hubei & $\mathrm{S} 183$ & 罗汉果 Luohanguo & 河北石家庄 Shijiazhuang, Hebei \\
\hline S081 & A1495-1 & 湖北武汉 Wuhan, Hubei & $\mathrm{S} 184$ & 中花 16 Zhonghua 16 & 河北石家庄 Shijiazhuang, Hebei \\
\hline S082 & A1495-2 & 湖北武汉 Wuhan, Hubei & S185 & 冀花 16 Jihua 16 & 河北石家庄 Shijiazhuang, Hebei \\
\hline S083 & A1497 & 湖北武汉 Wuhan, Hubei & ST01 & 中花 16 Zhonghua 16 & 湖北武汉 Wuhan, Hubei \\
\hline S084 & A1504-1 & 湖北武汉 Wuhan, Hubei & ST02 & 中花 21 Zhonghua 21 & 湖北武汉 Wuhan, Hubei \\
\hline S085 & A1504-2 & 湖北武汉 Wuhan, Hubei & ST03 & A1458-2 & 湖北武汉 Wuhan, Hubei \\
\hline S086 & A1504-3 & 湖北武汉 Wuhan, Hubei & ST04 & 宛花 2 号 Wanhua 2 & 湖北武汉 Wuhan, Hubei \\
\hline S087 & A1504-4 & 湖北武汉 Wuhan, Hubei & ST05 & 中花 15 Zhonghua 15 & 湖北武汉 Wuhan, Hubei \\
\hline S088 & A $1504-5$ & 湖北武汉 Wuhan, Hubei & ST06 & 中花 12 Zhonghua 12 & 湖北武汉 Wuhan, Hubei \\
\hline S089 & A1504-6 & 湖北武汉 Wuhan, Hubei & ST07 & 19A1385 & 湖北武汉 Wuhan, Hubei \\
\hline S090 & A1504-7 & 湖北武汉 Wuhan, Hubei & ST08 & 皖花 9 号 Wanhua 9 & 安徽合肥 Hefei, Anhui \\
\hline S091 & A $1507-1$ & 湖北武汉 Wuhan, Hubei & ST09 & 徐花甜 29 Xuhuatian 29 & 江苏徐州 Xuzhou, Jiangsu \\
\hline S092 & A1507-2 & 湖北武汉 Wuhan, Hubei & ST10 & 中花 9 号 Zhonghua 9 & 湖北武汉 Wuhan, Hubei \\
\hline S093 & A1507-4 & 湖北武汉 Wuhan, Hubei & ST11 & 远杂 9102 Yuanza 9102 & 江苏南京 Nanjing, Jiangsu \\
\hline S094 & A $1507-5$ & 湖北武汉 Wuhan, Hubei & ST12 & 宁泰 9922 Ningtai 9922 & 江苏南京 Nanjing, Jiangsu \\
\hline S095 & A1510-1 & 湖北武汉 Wuhan, Hubei & ST13 & A1447-6 & 湖北武汉 Wuhan, Hubei \\
\hline S096 & A $1510-2$ & 湖北武汉 Wuhan, Hubei & ST14 & 苏花 0537 Suhua 0537 & 江苏南京 Nanjing, Jiangsu \\
\hline S097 & A1513-1 & 湖北武汉 Wuhan, Hubei & ST15 & 大四粒红 Dasilihong & 湖北武汉 Wuhan, Hubei \\
\hline S098 & A1513-2 & 湖北武汉 Wuhan, Hubei & ST16 & 冀花 16 Jihua 16 & 湖北武汉 Wuhan, Hubei \\
\hline S099 & A1513-3 & 湖北武汉 Wuhan, Hubei & ST17 & 苏花 1713 Suhua 1713 & 江苏南京 Nanjing, Jiangsu \\
\hline $\mathrm{S} 100$ & A1513-4 & 湖北武汉 Wuhan, Hubei & ST18 & 冀花 11 Jihua 11 & 湖北武汉 Wuhan, Hubei \\
\hline S101 & A1513-5 & 湖北武汉 Wuhan, Hubei & ST19 & A1478-2 & 湖北武汉 Wuhan, Hubei \\
\hline S102 & A1513-6 & 湖北武汉 Wuhan, Hubei & ST20 & 徐花甜 30 Xuhuatian 30 & 江苏徐州 Xuzhou, Jiangsu \\
\hline $\mathrm{S} 103$ & A1513-7 & 湖北武汉 Wuhan, Hubei & & & \\
\hline
\end{tabular}




\section{2 研究方法}

\subsection{1 光谱采集采用美国 Unity 科技公司生产}

的 SpectraStar XL 近红外光谱仪采集光谱, 光谱仪 扫描波长范围为 1100 2500 nm。调整环境温度在 20
${ }^{\circ} \mathrm{C}$ 左右, 样品在 $20^{\circ} \mathrm{C}$ 左右恒温放置 $48 \mathrm{~h}$ 以上, 仪器 开机预热 $30 \mathrm{~min}$ 后, 每个样品取 15 20 粒籽仁, 装 入直径为 $3 \mathrm{~cm}$ 的小样品杯, 重复装样 3 次, 获得平 均光谱用于建模(图 1)。
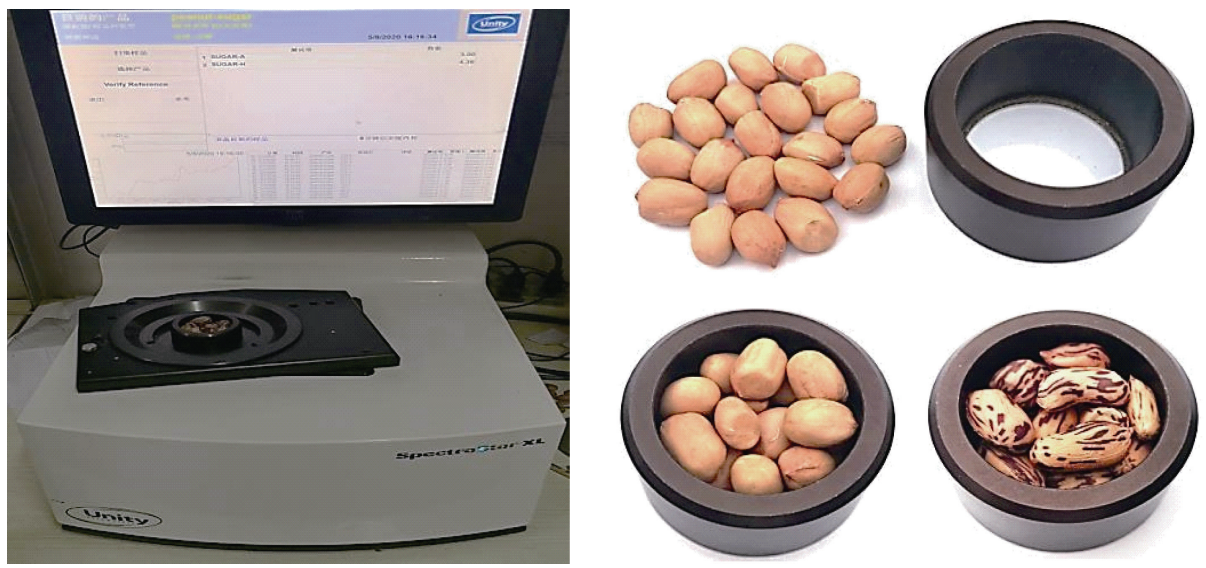

图 1 本研究所用的近红外仪及小样品杯

Fig. 1 Near infrared reflectance spectroscopy instrument Unity-SpectrastarXL and small cup used in this experiment

\subsection{2 蔗糖含量测定 将采集光谱所用的花生籽} 仁用磨样器磨碎, 称取 $1 \mathrm{~g}$ 粉末样品(重复 3 次), 至 $50 \mathrm{~mL}$ 的离心管中, 加入 $10 \mathrm{~mL}$ 的 $80 \%$ 乙醇溶液, $80^{\circ} \mathrm{C}$ 水浴 $30 \mathrm{~min}$; 冷却至室温, 取上清 $2 \mathrm{~mL}, 14,000 \times \mathrm{g}$ 离 心 $5 \mathrm{~min}$; 离心后取上清 $700 \mu \mathrm{L}$ ，过滤至上样瓶中待 上机检测。高效液相色谱型号为 Agilent 1290, 示差 折光检测器(RID)检测, 柱子型号 20RBAX NH2 (4.6 $\mathrm{mm} \times 250 \mathrm{~mm}, 5 \mu \mathrm{m})$, 流动相为 75：25 (v/v)乙腈/水 溶液, 流速 $1 \mathrm{~mL} \mathrm{~min}^{-1}$, 柱温 $40^{\circ} \mathrm{C}$, 进样量 $10 \mu \mathrm{L}$ 。 按照 GB 5009.8-2016 ${ }^{[22]}$ 中高效液相法的标准溶液配 制方法, 配制 2.0、4.0、6.0、8.0 和 $10.0 \mathrm{mg} \mathrm{mL}^{-1}$ 浓 度的标准溶液, 过滤至上样瓶进行上机测定, 记录 不同浓度标准溶液的峰面积, 以峰面积对浓度进行 线性回归, 构建标准曲线。试样溶液上机检测, 记录 目标峰面积, 从标准曲线中获得试样溶液中蔗糖的 浓度。

1.2.3 模型构建与优化采用Unity科技公司自 带的UCAL近红外定标软件构建模型, 将测得的蔗 糖含量化学值与采集的近红外光谱导入UCAL软件 进行拟合光谱处理，采用最小二乘法优化建模，建 模过程中自动剔除较大剩余值的异常样品, 然后再 进行内部交叉验证剔除异常值, 通过比较模型的决 定系数 $\left(R^{2}\right)$ 和标准差(RMSECV)衡量模型质量, 篮选 最佳模型, 并比较样品预测值与化学值的决定系数 $\left(R^{2}\right)$ 和均方差(Mean Square Deviation, MSD)来衡量 模型的质量。
1.2.4 模型的外部验证选取 20 个蔗糖含量显著 差异的花生品种(系), 利用建立的近红外方法检测 其蔗糖含量, 记录近红外模型的预测值, 再利用液 相色谱法(HPLC)分析样品的蔗糖含量, 比较NIR预 测值与HPLC测定化学值的相关性和准确性。

\subsection{5 高糖高油酸品系选育过程 2015年春季,} 以普通油酸花生品种“吉花02-1-4” (蔗糖含量为4.0\%) 为母本，高油酸品种“中花 26 ” (蔗糖含量 $2.3 \%$ ) 为父 本配制杂交组合，并收获 $\mathrm{F}_{1}$ 种子。2015年秋季，将 $\mathrm{F}_{1}$ 种植于广东湛江, 收获 $\mathrm{F}_{2}$ 种子。2016年春季, 使用近 红外检测单粒 $\mathrm{F}_{2}$ 种子中油酸含量, 选择油酸含量在 $75 \%$ 以上的种子，种植于湖北武汉，并收获 $\mathrm{F}_{3}$ 单株。 2017年春季, 使用近红外检测 $\mathrm{F}_{3}$ 单株种子油酸含量, 选择油酸含量在 $75 \%$ 以上的单株, 种植于湖北武汉, 收获 $\mathrm{F}_{4}$ 。2018年春季, 将收获的 $\mathrm{F}_{4}$ 按株系种植于武汉, 调查农艺性状, 收获 $\mathrm{F}_{5}, 2018$ 年冬季, 将 $\mathrm{F}_{5}$ 种植于海 南三亚, 收获 $\mathrm{F}_{6}$ 株系。2019年, 利用本研究建立的单 株蔗糖含量近红外模型测定 $\mathrm{F}_{6}$ 株系的蔗糖含量, 并 经液相色谱分析验证，获得蔗糖含量 $7 \%$ 以上、油酸 $78 \%$ 以上的优良株系。

\section{2 结果与分析}

\section{1 样品中蔗糖含量的化学分析结果}

采用高效液相色谱法测定了 185 份花生籽仁的蔗 糖含量, 样品的蔗糖测定值见表 2。蔗糖含量平均 $4.82 \%$, 变异范围 $1.02 \% \sim 8.48 \%$, 标准差为 1.96 , 标准 
误 0.011 , 变异系数 $40.66 \%$, 表明本试验选择花生材 料的蔗糖含量分布范围广, 变异系数大, 代表性好。

\section{2 近红外模型构建}

采集的 185 份花生籽仁近红外光谱如图 2 所示, 建模样品近红光谱曲线趋势大致相同，但不同样品 的吸收峰强度不同。表明花生小样品杯扫描获得的 近红外光谱图可以用于花生籽粒蔗糖含量的定量分
析。对花生中蔗糖的化学值和采集的近红外光谱数 据进行拟合光谱处理, 采用偏最小二乘法(PLS)的化 学计量学方法建立数学模型, 反复采用内部交叉验 证剔除异常值, 通过比较模型决定系数 $\left(R^{2}\right)$ 和均方 差(MSD)衡量模型质量，篮选最佳模型。去除异常值 后, 176 份样品建立模型的决定系数 $\left(R^{2}\right)$ 为 0.962 , 均 方差(MSD)为 0.383 (图 3)。

表 2 HPLC 法测定的定标样品蔗糖含量化学值

Table 2 Chemical values of sucrose content tested by HPLC

\begin{tabular}{|c|c|c|c|c|c|c|c|c|c|}
\hline $\begin{array}{c}\text { 样品编号 } \\
\text { Sample } \\
\text { ID }\end{array}$ & $\begin{array}{c}\text { 蔗糖含量 } \\
\text { Sucrose } \\
\text { content } \\
(\%) \\
\end{array}$ & $\begin{array}{c}\text { 样品编号 } \\
\text { Sample } \\
\text { ID }\end{array}$ & $\begin{array}{c}\text { 蔗糖含量 } \\
\text { Sucrose } \\
\text { content } \\
(\%) \\
\end{array}$ & $\begin{array}{c}\text { 样品编号 } \\
\text { Sample } \\
\text { ID }\end{array}$ & $\begin{array}{c}\text { 蔗糖含量 } \\
\text { Sucrose } \\
\text { content } \\
(\%) \\
\end{array}$ & $\begin{array}{c}\text { 样品编号 } \\
\text { Sample } \\
\text { ID }\end{array}$ & $\begin{array}{c}\text { 蔗糖含量 } \\
\text { Sucrose } \\
\text { content } \\
(\%) \\
\end{array}$ & $\begin{array}{c}\text { 样品编号 } \\
\text { Sample } \\
\text { ID }\end{array}$ & $\begin{array}{c}\text { 蔗糖含量 } \\
\text { Sucrose } \\
\text { content } \\
(\%) \\
\end{array}$ \\
\hline S001 & 6.43 & S038 & 7.22 & S075 & 5.82 & $\mathrm{~S} 112$ & 5.50 & S149 & 2.47 \\
\hline S002 & 7.66 & S039 & 7.02 & S076 & 6.25 & S113 & 6.50 & $\mathrm{~S} 150$ & 1.68 \\
\hline S003 & 5.46 & S040 & 6.91 & S077 & 5.61 & S114 & 3.95 & S151 & 2.07 \\
\hline S004 & 6.48 & S041 & 6.86 & S078 & 6.35 & S115 & 5.77 & S152 & 2.45 \\
\hline S005 & 5.93 & S042 & 6.66 & S079 & 5.68 & S116 & 5.77 & S153 & 1.75 \\
\hline S006 & 5.36 & S043 & 6.58 & S080 & 5.46 & S117 & 4.77 & S154 & 1.33 \\
\hline S007 & 6.17 & S044 & 6.43 & S081 & 6.12 & S118 & 5.68 & S155 & 2.10 \\
\hline S008 & 5.38 & S045 & 6.26 & S082 & 6.33 & S119 & 6.53 & S156 & 1.71 \\
\hline S009 & 5.05 & S046 & 6.04 & S083 & 6.47 & $\mathrm{~S} 120$ & 5.15 & S157 & 1.68 \\
\hline S010 & 6.51 & S047 & 5.97 & S084 & 5.59 & $\mathrm{~S} 121$ & 5.70 & S158 & 1.70 \\
\hline S011 & 6.67 & S048 & 6.42 & S085 & 5.45 & $\mathrm{~S} 122$ & 2.38 & S159 & 2.01 \\
\hline S012 & 6.77 & S049 & 8.48 & S086 & 5.74 & $\mathrm{~S} 123$ & 1.87 & $\mathrm{~S} 160$ & 2.12 \\
\hline S013 & 8.01 & S050 & 6.22 & S087 & 6.58 & $\mathrm{~S} 124$ & 1.68 & S161 & 2.80 \\
\hline S014 & 6.86 & S051 & 6.06 & S088 & 6.16 & $\mathrm{~S} 125$ & 2.13 & $\mathrm{~S} 162$ & 2.05 \\
\hline S015 & 5.85 & S052 & 5.86 & S089 & 6.45 & S126 & 2.16 & S163 & 2.72 \\
\hline S016 & 5.03 & S053 & 5.90 & S090 & 5.68 & $\mathrm{~S} 127$ & 1.65 & S164 & 4.00 \\
\hline S017 & 5.44 & S054 & 6.20 & S091 & 5.83 & $\mathrm{~S} 128$ & 2.09 & S165 & 3.52 \\
\hline S018 & 5.94 & S055 & 6.00 & S092 & 6.09 & S129 & 1.27 & S166 & 8.39 \\
\hline S019 & 6.20 & S056 & 6.24 & S093 & 7.48 & $\mathrm{~S} 130$ & 1.29 & S167 & 2.67 \\
\hline S020 & 6.31 & S057 & 6.58 & S094 & 7.55 & $\mathrm{~S} 131$ & 1.65 & S168 & 2.99 \\
\hline S021 & 4.94 & S058 & 5.44 & S095 & 6.56 & $\mathrm{~S} 132$ & 1.56 & S169 & 3.04 \\
\hline S022 & 5.23 & S059 & 5.30 & S096 & 7.32 & $\mathrm{~S} 133$ & 2.27 & $\mathrm{~S} 170$ & 3.00 \\
\hline S023 & 6.49 & S060 & 5.41 & S097 & 6.34 & S134 & 1.61 & S171 & 3.31 \\
\hline S024 & 5.24 & S061 & 5.31 & S098 & 6.16 & $\mathrm{~S} 135$ & 1.34 & $\mathrm{~S} 172$ & 2.80 \\
\hline S025 & 5.83 & S062 & 5.33 & S099 & 6.39 & $\mathrm{~S} 136$ & 1.87 & $\mathrm{~S} 173$ & 3.12 \\
\hline S026 & 4.93 & S063 & 6.34 & $\mathrm{~S} 100$ & 6.42 & S137 & 1.26 & S174 & 2.95 \\
\hline S027 & 5.53 & S064 & 5.42 & $\mathrm{~S} 101$ & 6.19 & S138 & 1.02 & S175 & 3.57 \\
\hline S028 & 6.20 & S065 & 6.49 & $\mathrm{~S} 102$ & 5.74 & S139 & 1.20 & S176 & 2.64 \\
\hline S029 & 5.07 & S066 & 5.93 & $\mathrm{~S} 103$ & 5.28 & $\mathrm{~S} 140$ & 2.32 & S177 & 2.21 \\
\hline S030 & 7.22 & S067 & 6.19 & S104 & 5.36 & S141 & 1.56 & S178 & 3.13 \\
\hline S031 & 7.36 & S068 & 6.67 & $\mathrm{~S} 105$ & 5.71 & $\mathrm{~S} 142$ & 2.36 & S179 & 3.96 \\
\hline S032 & 5.73 & S069 & 7.24 & S106 & 5.42 & S143 & 2.54 & S180 & 2.38 \\
\hline S033 & 5.85 & S070 & 6.59 & S107 & 5.91 & S144 & 1.36 & $\mathrm{~S} 181$ & 2.97 \\
\hline S034 & 8.06 & S071 & 6.56 & S108 & 5.89 & S145 & 1.52 & S182 & 3.50 \\
\hline S035 & 6.45 & $\mathrm{~S} 072$ & 5.28 & S109 & 6.36 & S146 & 2.22 & S183 & 2.89 \\
\hline S036 & 5.61 & S073 & 7.44 & S110 & 4.91 & S147 & 3.24 & S184 & 2.75 \\
\hline S037 & 5.60 & S074 & 6.41 & S111 & 5.22 & S148 & 2.50 & S185 & 3.17 \\
\hline
\end{tabular}




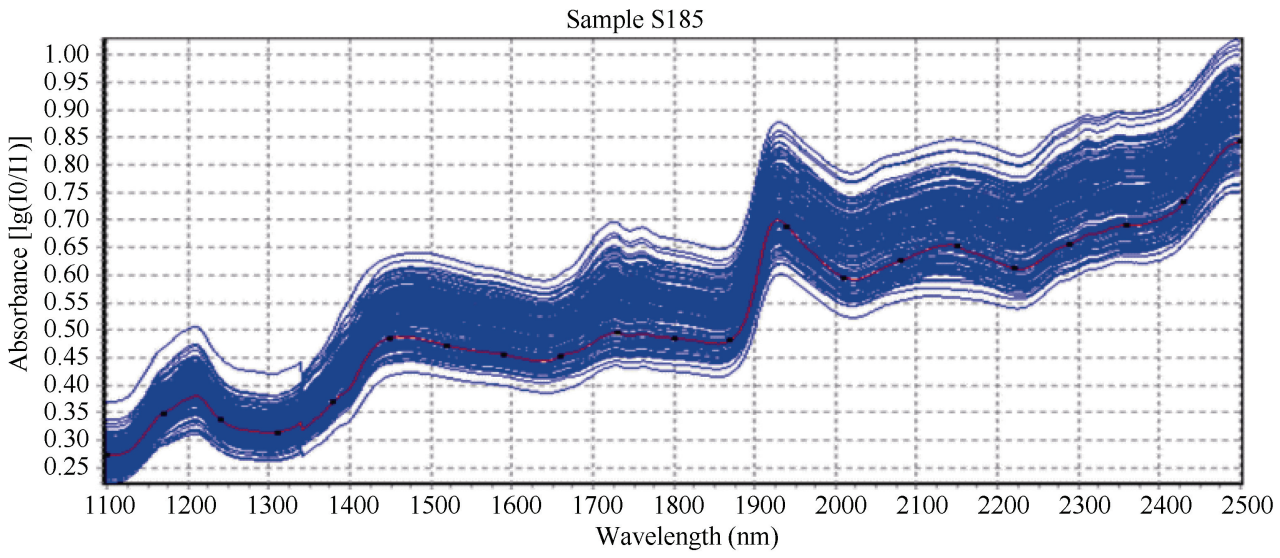

图 2 花生样品的近红外扫描光谱

Fig. 2 NIR spectrums of peanut kernel samples

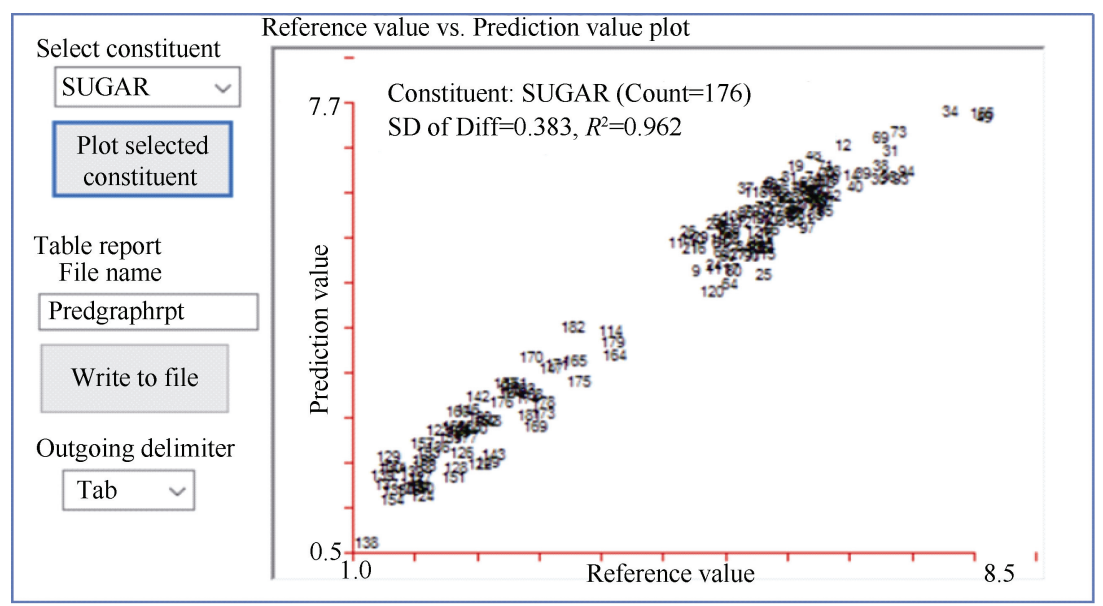

图 3 花生成熟籽仁蔗糖含量定标曲线决定系数

Fig. 3 Determination coefficient of predicted sucrose content in peanut seed by near-infrared reflectance spectroscopy

\section{3 模型的外部验证}

利用所建立的定标模型, 用近红外分析仪和化学 方法对 20 份没有参加定标的花生材料作为外部验证 样品集, 检验模型预测效果。熫糖含量的预测值与化
学值的绝对偏差在 $-1.44 \% \sim 0.72 \%$ 之间, 决定系数 $\left(R^{2}\right)$ $0.947, t=0.233<t_{0.05}=2.093$ (图 4), 相关性达到极显 著水平, 表明利用这个模型得到的预测结果准确, 具有 很高的可信度, 可用于花生籽仁熫糖含量的快速鉴定。

表 3 花生种子蔗糖含量近红外模型的验证

Table 3 Validation of near infrared model for sucrose content in peanut seed (\%)

\begin{tabular}{|c|c|c|c|c|c|c|c|}
\hline $\begin{array}{l}\text { 样品编号 } \\
\text { Sample ID }\end{array}$ & $\begin{array}{c}\text { 化学值 } \\
\text { Chemical value }\end{array}$ & $\begin{array}{c}\text { 预测值 } \\
\text { Prediction value } \\
\end{array}$ & $\begin{array}{c}\text { 偏差 } \\
\text { Deviation } \\
\end{array}$ & $\begin{array}{l}\text { 样品编号 } \\
\text { Sample ID }\end{array}$ & $\begin{array}{c}\text { 化学值 } \\
\text { Chemical value }\end{array}$ & $\begin{array}{c}\text { 预测值 } \\
\text { Prediction value } \\
\end{array}$ & $\begin{array}{c}\text { 偏差 } \\
\text { Deviation }\end{array}$ \\
\hline ST01 & 0.780 & 1.263 & 0.483 & ST11 & 2.220 & 2.563 & 0.343 \\
\hline ST02 & 0.818 & 1.256 & 0.438 & ST12 & 2.320 & 2.810 & 0.490 \\
\hline ST03 & 9.030 & 7.590 & -1.440 & ST13 & 6.520 & 6.152 & -0.368 \\
\hline ST04 & 0.940 & 1.174 & 0.234 & ST14 & 2.570 & 3.290 & 0.720 \\
\hline ST05 & 1.021 & 1.234 & 0.213 & ST15 & 2.910 & 2.696 & -0.214 \\
\hline ST06 & 1.078 & 0.716 & -0.362 & ST16 & 3.020 & 3.730 & 0.710 \\
\hline ST07 & 1.119 & 1.164 & 0.045 & ST17 & 3.370 & 3.213 & -0.157 \\
\hline ST08 & 1.246 & 0.927 & -0.320 & ST18 & 3.540 & 2.982 & -0.558 \\
\hline ST09 & 4.773 & 5.268 & 0.495 & ST19 & 4.045 & 4.518 & 0.473 \\
\hline ST10 & 1.851 & 2.246 & 0.395 & ST20 & 5.206 & 4.706 & -0.500 \\
\hline
\end{tabular}




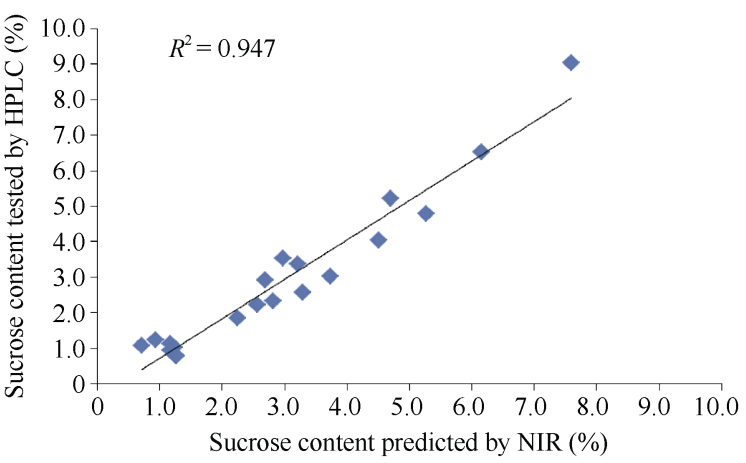

图 4 外部验证花生样品的蔗糖含量与测定化学值的相关性

Fig. 4 Correlation coefficients of sucrose content between HPLC and NIR testing in the external peanut samples

\section{4 模型在高含糖量、高油酸优良品系中的应用} 效果

2019年利用本研究建立的单株蔗糖含量近红外 模型，测定了“吉花02-1-4×中花26”的168个 $\mathrm{F}_{6}$ 株系 的蔗糖含量，并经液相色谱分析，获得蔗糖含量 $6.8 \%$ 以上、油酸 $78 \%$ 以上的优良株系(表 3 )。从表 3 可以看出，获得的高蔗糖材料的含油量均低于 $48 \%$, 最低的仅 $42 \%$ ，属于低油品系，油酸含量均在 $78 \%$ 以 上，属于高油酸材料。从图5可以看出，本研究篮选
出的6份高蔗糖、低含油量、高油酸品系在荚果大小、 每荚果粒数、荚果和籽仁形状等食用品质相关的指 标上也有很大差别，具有培育成不同食用型花生品 种的潜力。

\section{3 讨论}

近年来，花生油用和食用品质及其遗传改良受 到越来越多的关注, 品质指标从最初的含油量、蛋白 质含量逐步扩展到脂肪酸、氨基酸、蔗糖含量、白藜 芦醇含量等。虽然这些品质指标均建有精准的化学测 定方法, 但普遍费时费力, 因而推动了近红外技术在 花生品质改良上的应用，针对不同的近红外仪型号， 国内建立了多个品质性状的近红外模型 ${ }^{[13-14,16,18]}$ 。同 时，模型检测需要的花生籽仁量越来越少，甚至建 立了单粒籽仁主要脂肪酸含量的近红外模型 ${ }^{[15,19]}$, 模型预测结果的准确性也越来越好，显著提升了品 质育种效率。本研究利用美国Unity公司的近红外仪, 建立了小样品杯的蔗糖含量预测模型, 能对杂交后 代早期的单株籽仁进行检测, 并具有较好的预测结 果, 是对已有蔗糖含量模型的改进和丰富 ${ }^{[20-21]}$ 。为更 好地进行蔗糖含量的预测和种子纯度分析，在以后

表 4 获得的高蔗糖高油酸花生品系的主要性状

Table 4 Main characters of selected peanut lines with high sucrose and oleic acid

\begin{tabular}{|c|c|c|c|c|c|c|c|c|}
\hline $\begin{array}{c}\text { 品系名称 } \\
\text { Lines } \\
\text { name }\end{array}$ & $\begin{array}{c}\text { 蔗糖含量 } \\
\text { Sucrose } \\
\text { content (\%) }\end{array}$ & $\begin{array}{c}\text { 含油量 } \\
\text { Oil content } \\
(\%) \\
\end{array}$ & $\begin{array}{c}\text { 油酸 } \\
\text { Oleate } \\
\text { content (\%) }\end{array}$ & $\begin{array}{c}\text { 百果重 } \\
\text { 100-pod } \\
\text { weight (g) }\end{array}$ & $\begin{array}{c}\text { 百仁重 } \\
\text { 100-seed } \\
\text { weight (g) }\end{array}$ & $\begin{array}{c}\text { 出仁率 } \\
\text { Shelling } \\
\text { percentage }(\%)\end{array}$ & $\begin{array}{c}\text { 荚果长 } \\
\text { Pod length } \\
(\mathrm{cm}) \\
\end{array}$ & $\begin{array}{c}\text { 籽仁长 } \\
\text { Seed length } \\
(\mathrm{cm}) \\
\end{array}$ \\
\hline SYT5-1 & 8.45 & 46.8 & 79.6 & 212 & 67 & 76.4 & 5.1 & 1.6 \\
\hline SYT7-1 & 7.63 & 45.3 & 80.2 & 204 & 56 & 76.6 & 4.6 & 1.5 \\
\hline SYT3-1 & 7.12 & 44.6 & 78.6 & 162 & 76 & 70.3 & 3.9 & 1.8 \\
\hline SYT3-2 & 8.02 & 47.8 & 81.5 & 176 & 80 & 74.9 & 3.7 & 1.8 \\
\hline SYT4-1 & 7.88 & 45.3 & 79.8 & 159 & 67 & 76.7 & 3.0 & 1.5 \\
\hline SYT4-2 & 9.07 & 42.2 & 81.1 & 168 & 80 & 78.6 & 3.2 & 1.5 \\
\hline
\end{tabular}
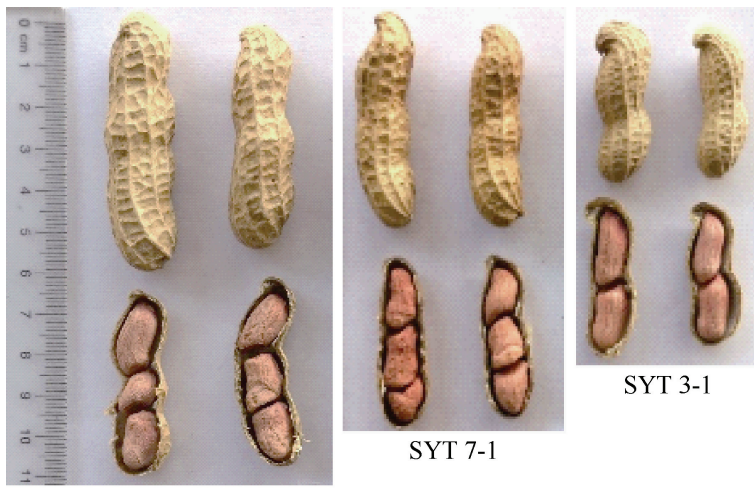

SYT 3-1
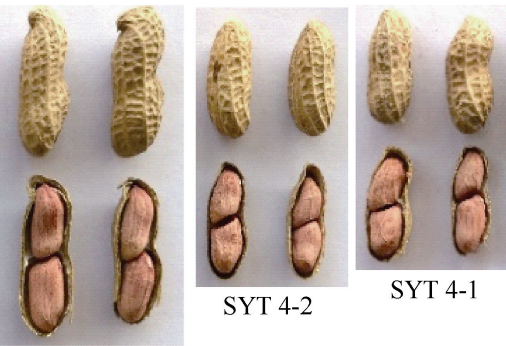

SYT 4-2
SYT 4-1

图 5 获得的高糖高油酸优良花生品系

Fig. 5 Selected peanut lines with high sugar and high oleic acid content 
的工作中, 还需要建立单粒花生蔗糖含量的近红外 模型。

利用一个近红外仪对多个品质指标进行跟踪检 测已经成为可能。如本研究就同时利用了含油量、 油酸含量、含糖量 3 个近红外检测模型对同一样品 进行检测, 获得了兼具高含糖量、低含油量、高油 酸的优良食用型花生新品系, 这得益于当前花生重 要品质参数近红外模型越来越丰富。随着花生品质 研究的深入，一些含量不高、目前关注较少、具有 重要价值的品质性状也需要逐步建立近红外预测 模型。

本研究在 2 个蔗糖含量并不很高的花生品种吉 花 02-1-4 (4.0\%)和中花 26 (2.3\%)的杂交后代中篮选 出一批含糖量超过 7\%的优良品系，蔗糖含量表现出 明显的超亲现象，推测可能的原因是不同材料中控 制蔗糖含量的位点不同，杂交聚合产生了超亲效应 所致。同时, 我们也看到, 熫糖含量高的品系普遍含 油量显著降低, 说明这一杂交组合中产生蔗糖含量 超亲的后代与含油量合成受阻直接或间接相关，因 此，深入研究高糖、低油品系形成的分子基础也十 分必要。另外, 虽然成熟花生种仁的蔗糖含量与总 含糖量及甜度相关，但成熟花生种仁中还存在葡萄 糖、果糖等其他糖类以及影响甜度的单宁等苦味成 分, 这些成分对甜度亦有一定的正向和负向影响, 食用型花生的甜味及适口性评价仍需在蔗糖含量测 定的基础上，由经过训练的人员进行描述性感官风 味分析 ${ }^{[3,5]}$ 。

\section{4 结论}

本研究利用籽仁蔗糖含量差异显著的花生材料, 构建了花生籽仁蔗糖含量的小样品杯近红外定标模 型, 模型的决定系数 $R^{2}=0.962$, 验证样品集的决定 系数为 $R^{2}=0.947$, 可以较好地预测花生籽仁的蔗糖 含量。利用该模型配合含油量、油酸含量近红外模 型，高效的在“吉花 02-1-4×中花 26”杂交后代群体中 发掘出 6 份高熫糖、低油、高油酸、农艺性状优良 的食用型花生新品系。

\section{References}

[1] 廖伯寿, 殷艳, 马霓. 中国油料作物产业发展回顾与展望. 农 学学报, 2018, 8(1): 107-112.

Liao B S, Yin Y, Ma N. Review and future prospects of oil crops industry development in China. J Agric, 2018, 8(1): 107-112 (in Chinese with English abstract).

[2] 房元瑾, 孙子淇, 苗利娟, 齐飞艳, 黄冰艳, 郑峥, 董文召,
汤丰收, 张新友. 花生种仁外观和营养品质特征及食用型花 生育种利用分析. 植物遗传资源学报, 2018, 19: 875-886.

Fang Y J, Sun Z Q, Miao L J, Qi F Y, Huang B Y, Zheng Z, Dong W Z, Tang F S, Zhang X Y. Characterization of kernel appearance and nutritional quality in peanut accessions and its application for food-use peanut breeding. J Plant Genet Resour, 2018, 19: 875-886 (in Chinese with English abstract).

[3] Pattee H E, Isleib T G, Giesbrecht F G. Variation in intensity of sweet and bitter sensory attributes across peanut genotypes. Peanut Sci, 1998, 25: 63-69.

[4] Pattee H, Isleib T, Giesbrecht F, McFeeters R. Investigations into genotypic variations of peanut carbohydrates. J Agric Food Chem, 2000, 48: 750-756.

[5] Pattee H E, Isleib T G, Giesbrecht F G, McFeeters R F. Relationships of sweet, bitter, and roasted peanut sensory attributes with carbohydrate components in peanuts. J Agric Food Chem, 2000, 48: 757-763.

[6] Isleib T, Pattee H, Giesbrecht F. Oil, sugar, and starch characteristics in peanut breeding lines selected for low and high oil content and their combining ability. J Agric Food Chem, 2004, 52: 3165-3168.

[7] Bishi S K, Kumar L, Dagla M C, Mahatma M K, Rathnakumar A L, Lalwani H B. Characterization of Spanish peanut germplasm (Arachis hypogaea L.) for sugar profiling and oil quality. Ind Crops Prod, 2013, 51: 46-50.

[8] Bishi S K, Lokesh K, Mahatma M K, Khatediya N, Chauhan S M, Misra J B. Quality traits of Indian peanut cultivars and their utility as nutritional and functional food. Food Chem, 2015, 67: 107-114.

[9] 王铎, 张力, 范素杰, 韩笑, 吴晗, 王丕武, 姚丹, 张君. 大豆 主要脂肪酸含量近红外模型的建立. 大豆科学, 2017, 36: 295-299.

Wang D, Zhang L, Fan S J, Han X, Wu H, Wang P W, Yao D, Zhang J. Establishment of NIRS model for the main fatty acids in soybean. Soybean Sci, 2017, 36: 295-299 (in Chinese with English abstract).

[10] 康月琼, 郝风, 柴勇, 杨俊英, 黄永东, 熊英, 褚能明, 余官 平. 油菜品质近红外检测模型建立的研究. 中国农学通报, 2011, 27(5): 144-148.

Kang Y Q, Hao F, Cai Y, Yang J Y, Huang Y D, Xiong Y, Chu N M, Yu G P. Study on construction of determination model of rapeseed quality with near-infrared spectroscopy. Chin Agric Sci Bull, 2011, 27(5): 144-148 (in Chinese with English abstract).

[11] 刘盼, 张艳欣, 黎冬华, 王林海, 高媛, 周瑢, 张秀荣, 魏 金金. 基于近红外模型的芝麻核心种质油脂和蛋白质含量变异 分析. 中国油料作物学报, 2016, 38: 722-729.

Liu P, Zhang Y X, Li D H, Wang L H, Gao Y, Zhou R, Zhang X R, Wei X. Oil and protein contents analysis of sesame core collections based on near infrared reflectance spectroscopy model. Chin J Oil Crop Sci, 2016, 38: 722-729 (in Chinese with English abstract).

[12] Misra J B, Mathur R S, Bhatt D M. Near-infrared transmittance spectroscopy: a potential tool for non-destructive determination of oil content in groundnuts. J Sci Food Agric, 2000, 80: $237-240$.

[13] 曲艺伟, 张鹤, 韩笑, 李雪䒯, 王传堂, 王丕武, 姚丹, 张君. 
花生脂肪酸近红外模型的建立. 分子植物育种, 2019, 17: $568-578$.

Qu Y W, Zhang H, Han X, Li X Y, Wang C T, Wang P W, Yao D, Zhang J. Establishment of near-infrared model of peanut fatty acids. Mol Plant Breed, 2019, 17: 568-578 (in Chinese with English abstract).

[14] Fox G, Cruickshank A. Near infrared reflectance as a rapid and inexpensive surrogate measure for fatty acid composition and oil content of peanuts (Arachis hypogaea L.). J Near Infrar Spec, 2005, 13: 287.

[15] Tillman B L, Gorbet D W, Person G. Predicting oleic and linoleic acid content of single peanut seeds using near-infrared reflectance spectroscopy. Crop Sci, 2006, 46: 2121-2126.

[16] 王秀贞, 唐月异, 张建成, 崔凤高, 陈殿绪, 迟玉成, 王传堂. 近红外技术分析化学诱变对花生脂肪、蛋白质和蔗糖含量的 影响. 花生学报, 2009, 38(4): 5-8.

Wang X Z, Tang Y Y, Zhang J C, Cui F G, Chen D X, Chi Y C, Wang C T. Analyzing the effect of chemical treatment on oil, protein and sucrose content of peanut with NIRS. J Peanut Sci, 2009, 38(4): 5-8 (in Chinese with English abstract).

[17] Wang C T, Tang Y Y, Wang X Z, Chen D X, Yu S L. Evaluation of groundnut genotypes from China for quality traits. J Agric Res, 2011, 12: 1-5.

[18] 张建成, 王传堂, 王秀贞, 唐月异, 张树伟, 李贵杰. 花生自 然风干种子油酸、亚油酸和棕㭣酸含量的近红外分析模型构 建. 中国农学通报, 2011, 27(3): 90-93.

Zhang J C, Wang C T, Wang X Z, Tang Y Y, Zhang S W, Li G J. NIRS calibration models predictive of oleic, linoleic and palmitic acid content in sun-dried bulk peanut seed Samples. Chin Agric Sci Bull, 2011, 27(3): 90-93 (in Chinese with English abstract).
[19] 李建国, 薛晓梦, 张照华, 王志慧, 晏立英, 陈玉宁, 万丽云, 康彦平, 淮东欣, 姜慧芳, 雷永, 廖伯寿. 单粒花生主要脂肪 酸含量近红外预测模型的建立及其应用. 作物学报, 2019, 45: 1891-1898.

Establishment and applicant of near-infrared reflectance spectroscopy models for predicting main fatty acid contents of single seed in peanut. Acta Agron Sin, 2019, 45: 1891-1898 (in Chinese with English abstract).

[20] 秦利, 刘华, 杜培, 董文召, 黄冰艳, 韩锁义, 张忠信, 齐飞艳, 张新友. 基于近红外光谱法的花生种仁中蔗糖含量的测定. 中国油料作物学报, 2016, 38: 666-671.

Qin L, Liu H, Du P, Dong W Z, Huang B Y, Han S Y, Zhang Z X, Qi F Y, Zhang X Y. Determination of sucrose content in peanut seed kernel based on near infrared spectroscopy. Chin J Oil Crop Sci, 2016, 38: 666-671 (in Chinese with English abstract).

[21] 唐月异, 王秀贞, 刘婷, 吴琪, 孙全喜, 王志伟, 张欣, 王传堂, 邵俊飞. 花生自然风干种子蔗糖含量近红外定量分析模型构 建. 山东农业科学, 2018, 50(6): 159-162.

Tang Y Y, Wang X Z, Liu T, Wu Q, Sun Q X, Wang Z W, Zhang X, Wang C T, Shao J F. A near infrared spectroscopy model for predicting sucrose content of sun-dried peanut seeds. Shandong Agric Sci, 2018, 50(6): 159-162 (in Chinese with English abstract).

[22] 中华人民共和国卫生和计划生育委员会. GB 5009.8-2016. 食 品安全国家标准一一食品中果糖、葡萄糖、蔗糖、麦芽糖、乳 糖的测定. 北京: 中国标准出版社, 2016. pp 1-16.

National Health and Family Planning Commission of People's Republic of China. GB 5009. 8-2016. National Standards for Food Safety Determination of Fructose, Glucose, Sucrose, Maltose and Lactose Content in Food. Beijing: Standards Press of China, 2016. pp 1-16. 